\title{
Article
}

\section{Dynamic Viscoelastic Properties of Multi-Arm Star Polystyrenes in the Molten State}

\author{
Takashi Yamamoto, Yasuhiko OHTA, Toshikazu TAKIGAwA, and Toshiro MAsudA \\ Department of Material Chemistry, Graduate School of Engineering, Kyoto University, \\ Sakyo-ku, Kyoto 606-8501, Japan \\ (Received: July 12, 2001)
}

\begin{abstract}
Multi-arm star polystyrenes with various lengths and number of arms were synthesized, and their dynamic viscoelastic properties were investigated in the molten state. The master curves of the storage modulus for the multi-arm stars showed a shoulder at low frequencies, suggesting that a new mechanical relaxation exists at the long time scales after completion of entanglement relaxation. The enhancement of the zero-shear viscosity compared with the regular star polymers was also observed for the multi-arm star polymers.
\end{abstract}

Key words: Multi-arm star / Dynamic viscoelasticity / Long-time relaxation / Viscosity enhancement

\section{INTRODUCTION}

A regular star polymer, a star-shaped polymer with relatively small number of arms, is typical of branched polymers. Rheological properties of the regular star polymers have been studied by experiment ${ }^{1-3)}$ as well as theoretical approach. ${ }^{4)}$ It is well known that the entanglement relaxation of the regular star polymers is not affected by the number of arms but is strongly affected by the molecular weight of the arm.

In contrast, multi-arm star polymers consist of many arms, typically more than ten arms. These polymers are considered to have a kind of core-shell structure, due to high chain density at the center of the molecule. Previously, we have studied the rheological properties of multi-arm star polystyrenes in the molten state. ${ }^{5-6)}$ However, the information on the long-time relaxation behavior obtained was not so enough and accurate, primarily because the performance of the rheometer used was not so high comparing with up-to-date rheometers now available.

Multi-arm star polymers have been classified as an ultra-soft colloid system, and their unique dynamics has been reported. ${ }^{7}$ The structure and properties of the multi-arm star polymers were studied by scattering methods ${ }^{7-11)}$ and computer simulation. ${ }^{10,11)}$ These studies revealed that the polymer systems show a liquid-like ordering. In addition, the systems showed a new mechanical relaxation in the long time region. ${ }^{9-11)}$ This relaxation is believed to be due to the diffusional motion of the star cores back to the equilibrium state after deformation.
However details of the mechanical relaxation are still unclear at present. To investigate the new mechanical relaxation, the difference in viscoelasticity between the regular and multi-arm stars must be again examined more clearly.

In this study, we synthesized multi-arm star polystyrenes with various lengths and number of arms, and examined the dynamic viscoelasticity of the melts of these multi-arm star polystyrenes.

\section{EXPERIMENTAL}

Four-types of multi-arm star polystyrenes differing in length and/or number of arms were synthesized by a two-step preparation method. The sample code is listed in Table I. First, linear polystyrene (PS) was prepared by an anionic polymerization method in the mixture of benzene and diethylether using $s e c$-butyllithium as an initiator at $0^{\circ} \mathrm{C}$ for $24 \mathrm{hr}$. Then, a small amount of divinylbenzene (DVB) was added into the solution to couple the active ends. Before the coupling reaction, a small amount of polystyryl anion was recovered and terminated to determine the molecular weights of the arm (precursor). The coupling reaction was continued with stirring the solution for $c a$. 10 days at room temperature. Finally, the product was terminated and purified with methanol. To remove a small amount of lower and higher molecular weight fractions, the product was fractionated from benzene/methanol mixtures. Gel permeation chromatogram (GPC) of the samples was obtained with a Tosoh CCP and 
Table I Molecular characteristics and zero-shear viscosity $\eta_{0}$ of star polystyrenes.

\begin{tabular}{lcccccccc}
\hline & \multicolumn{3}{c}{ arm } & \multicolumn{3}{c}{ star } & $\mathrm{P}^{*}$ & $\eta_{0}$ \\
& $M_{\mathrm{w}} / 10^{4}$ & $M_{\mathrm{n}} / 10^{4}$ & $M_{\mathrm{w}} / M_{\mathrm{n}}$ & $M_{\mathrm{w}} / 10^{5}$ & $M_{\mathrm{n}} / 10^{5}$ & $M_{\mathrm{w}} / M_{\mathrm{n}}$ & & $/ 10^{5} \mathrm{Pas}$ \\
\hline LM23 & 5.68 & 5.33 & 1.07 & 9.77 & 9.23 & 1.06 & 17.3 & 10.5 \\
LM24 & 4.50 & 4.45 & 1.01 & 7.54 & 6.96 & 1.08 & 15.6 & 4.67 \\
LM26 & 2.57 & 2.37 & 1.09 & 10.4 & 8.76 & 1.18 & 36.9 & 0.96 \\
LM27 & 2.64 & 2.45 & 1.08 & 6.50 & 5.51 & 1.18 & 22.5 & 0.28
\end{tabular}

${ }^{*}$ Number of arms defined as a ratio of $M_{\mathrm{n}}$ of a star to that of an arm.

8020 series with a low-angle laser light scattering apparatus (LALLS, Tosoh LS-8000) to determine the molecular weights. Elute solvent was tetrahydrofuran (THF).

Dynamic viscoelasticity of the melts of the star samples was measured by using a Rheometric Scientific ARES rheometer with a cone-plate geometry $(25 \mathrm{~mm} \phi$, cone angle $0.04 \mathrm{rad})$. Before the measurement, the powder samples were molded into disk-shape specimens, and these disks were dried at $80^{\circ} \mathrm{C}$ in vacuo to prevent bubbling. Measurements were performed under nitrogen atmosphere to prevent degradation. The frequency dependence curves of the dynamic storage modulus $\left(G^{\prime}\right)$ and loss modulus ( $\left.G^{\prime \prime}\right)$ were obtained at several temperatures. To construct the master curves, the curves measured at each temperature were shifted horizontally as well as vertically. The reference temperature was set to be $160^{\circ} \mathrm{C}$.

\section{RESULTS AND DISCUSSION}

\subsection{Molecular Characterization}

A typical GPC profile of a multi-arm star polystyrene, LM23, is shown in Fig.1. The solid and dotted curves represent signals from RI and LS detectors, respectively. Both curves show a single peak. The other multi-arm star polymers also showed similar GPC profiles. Table I lists the numberaverage molecular weight $\left(M_{\mathrm{n}}\right)$, the weight-average molecular weight $\left(M_{\mathrm{w}}\right)$, and the polydispersity index $\left(M_{\mathrm{w}} / M_{\mathrm{n}}\right)$ calculated from the GPC curves using both RI and LS signals. The values of $M_{\mathrm{n}}, M_{\mathrm{w}}$ and $M_{\mathrm{w}} / M_{\mathrm{n}}$ for the multi-arm star polymers as well as for the precursor arms are listed in Table I. The number of arms $(P)$, defined as a ratio of $M_{\mathrm{n}}$ of a star to that of an arm, is also summarized in the table.

The number of arms changes the core size of a multi-arm star polymer with a core-shell structure. From Table I, the arms of LM23 and LM24 are about twice longer than those of LM26 and LM27. LM23 and LM24 have roughly the same number of arms as LM27. LM26 has almost the same length of arm as LM27, but the number of arms for LM26 is about 1.5 times more than that for LM27.

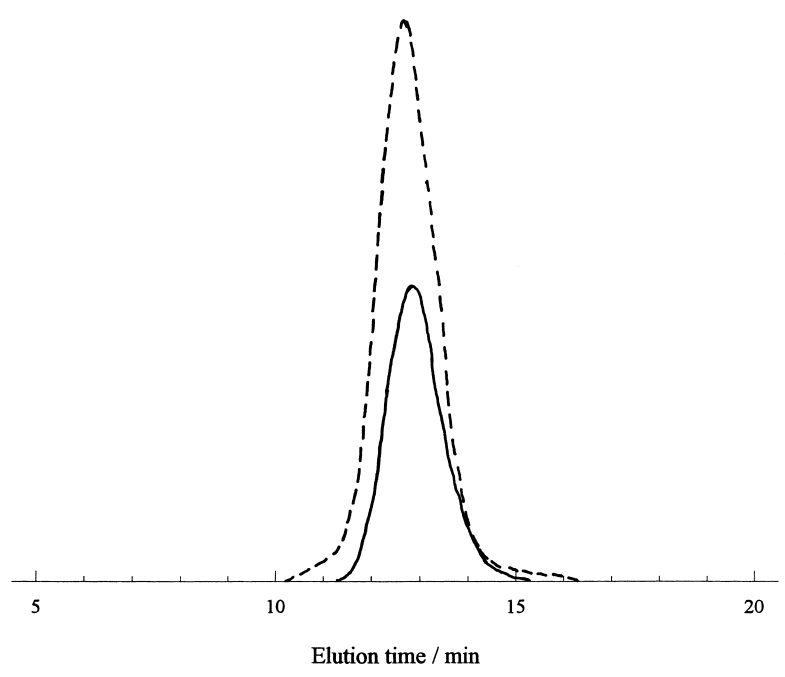

Fig.1 GPC Profile of LM23. The solid and dotted curves represent the signals of refractive index (RI) and light scattering (LS) detectors, respectively.

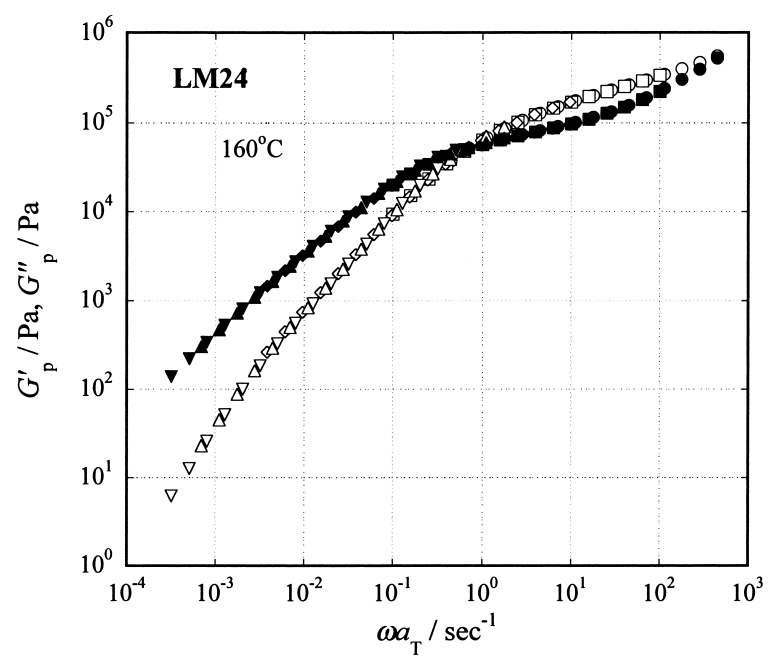

Fig.2 The frequency dependence of the dynamic storage modulus $\left(G^{\prime}\right)$ and loss modulus $\left(G^{\prime \prime}\right)$ for LM24 reduced at $160^{\circ} \mathrm{C}$. Unfilled and filled symbols correspond to $G^{\prime}$ and $G^{\prime}$, respectively.

\subsection{Dynamic Viscoelasticity}

The frequency dispersion curves of $G$ ' and $G$ ' for the multiarm stars were well superimposed to give the master curves, implying that the star polymers are thermorheologically simple. The values of the horizontal shift factor $a_{\mathrm{T}}$ were almost the same for all star samples and obeyed the WLF-type 


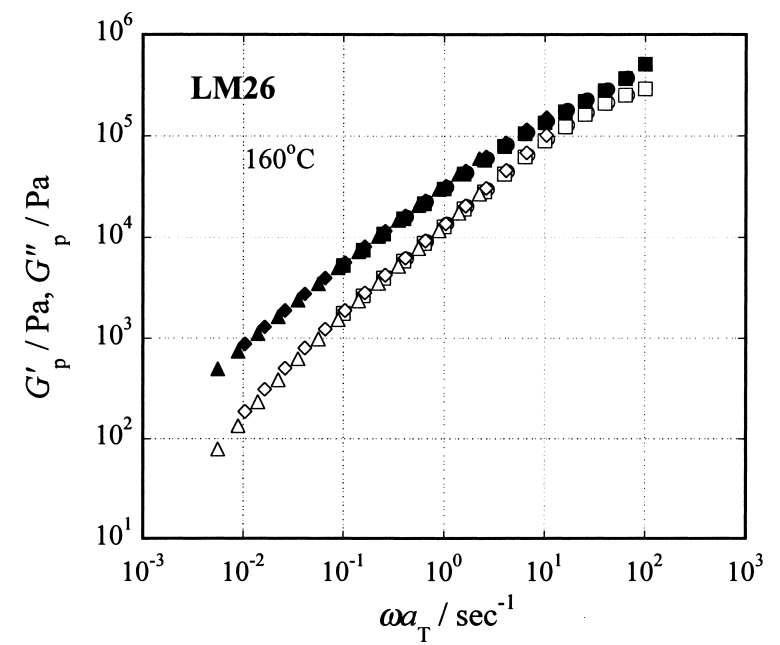

Fig.3 The frequency dependence of the dynamic storage modulus $\left(G^{\prime}\right)$ and loss modulus $\left(G^{\prime \prime}\right)$ for LM26 reduced at $160^{\circ} \mathrm{C}$. Unfilled and filled symbols correspond to $G^{\prime}$ and $G^{\prime \prime}$, respectively

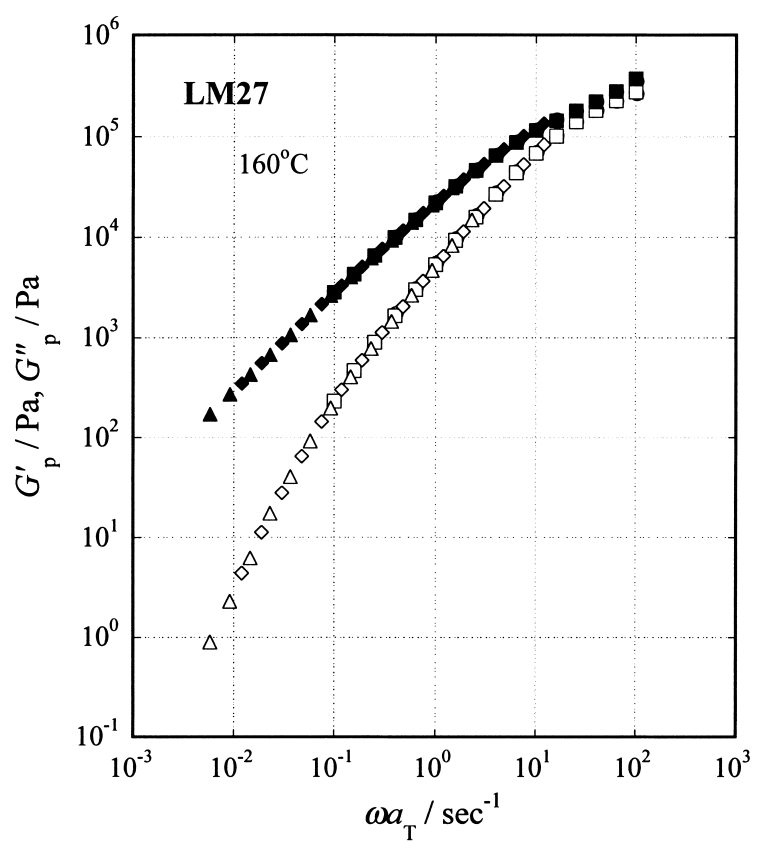

Fig.4. The frequency dependence of the dynamic storage modulus $\left(G^{\prime}\right)$ and loss modulus (G”) for LM27 reduced at $160^{\circ} \mathrm{C}$. Unfilled and filled symbols correspond to $G$ ' and $G$ ', respectively

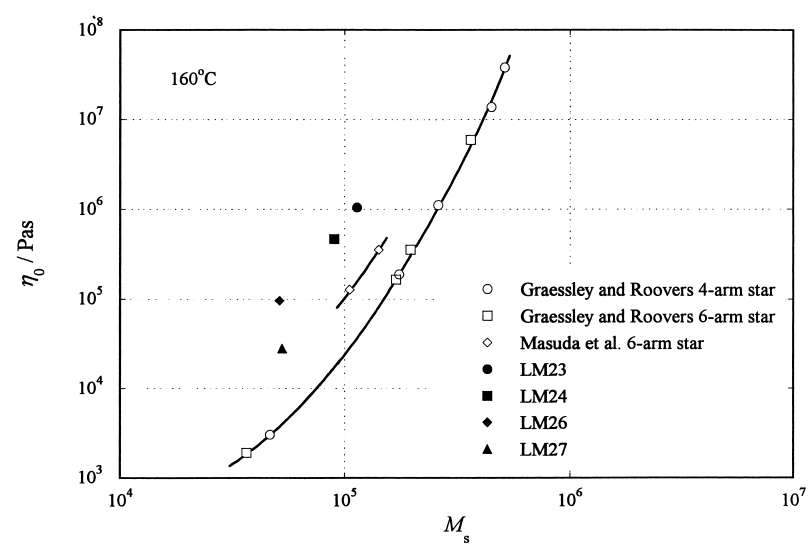

Fig.5 The plots of the zero-shear viscosity $\left(\eta_{0}\right)$ vs. span molecular weight $\left(M_{\mathrm{s}}\right)$ at $160^{\circ} \mathrm{C}$. equation. The master curves of $G$ ' and $G$ " for LM24 are shown in Fig.2. The master curves for LM23 were very similar to those for LM24, although we do not show the curves here.

In Fig.2, the transition zone, rubbery-plateau zone, and flow zone are seen in the reduced frequency range examined. The $G$ ' curve in the flow zone shows the relation $G^{\prime} \propto \omega$, giving the zero-shear viscosity $\left(\eta_{0}=\left[G^{\prime \prime} / \omega\right]_{\omega \rightarrow 0}\right) .{ }^{12)}$ The value of $\eta_{0}$ for LM24 is listed in Table I.

Figure 2 also demonstrates that a shoulder is observed in the $G$ ' curve at low frequencies where the entanglement relaxation is completed. The existence of this shoulder suggests that a new mechanical relaxation occurs in the long time region.

The master curves for LM26 and LM27 are shown in Figs.3 and 4, respectively. Both samples show the flow behavior characterized by the relation $G " \propto \omega$ at low frequencies. However, a distinct rubbery-plateau region cannot be observed for LM26 and LM27, as different from the behavior of LM24. Whether or not the entanglements actually exist in the system primarily depends on the molecular weight of the $\operatorname{arm}\left(M_{\mathrm{a}}\right)$, or equivalently, the span molecular weight $\left(M_{\mathrm{s}} ; M_{\mathrm{s}}=2 M_{\mathrm{a}}\right)$ of the star samples. The values of $M_{a}$ for LM23 and LM24 are larger than those of LM26 and LM27, as can be seen from Table I. Absence of the plateau region for LM26 and LM27 may indicate that these samples are non-entangled, while LM23 and LM24 are entangled, as long as judging from the $G$ ' and $G$ " curves, although the sensitivity to the existence of entanglements, of course, depends on features of the physical quantity measured. Even for the non-entangled systems, LM26 and LM27, a shoulder is also found in the $G$ ' curve at low frequencies. This shoulder must have the same origin of the relaxation as that for LM24. The long-time relaxation of the multi-arm star polystyrenes appears to be independent of whether or not the systems have entanglements.

Figure 5 shows the plots of $\eta_{0}$ against $M_{\mathrm{s}}$ for multi-arm stars investigated in this study. The figure also contains the data for regular stars ${ }^{2,3)}$ reported in literature. The $\eta_{0}$ data for the regular (4- and 6-arm) stars reported by Graessley and Roovers $^{2)}$ fall on a single curve, and these $\eta_{0}$ data increase exponentially with increasing $M_{\mathrm{s}}$. This is in good agreement with the experimental results reported by Fetters et al. ${ }^{1)}$ that $\eta_{0}$ is independent of the number of arms. The $\eta_{0}$ data for 6-arm stars obtained from our previous work ${ }^{3)}$ do not fall on the curve obtained from the data of Graessley and Roovers. The reason for this deviation is not clear, although the deviation might originate partly from the difference in $a_{\mathrm{T}}$

Figure 5 clearly shows that the viscosity of our multi-arm stars is enhanced compared with the regular stars. A 
comparison of $\eta_{0}$ of LM26 and LM27 reveals an interesting result: $\eta_{0}$ appears to increase as the number of arms increases, although LM26 and LM27 have the same $M_{\mathrm{a}}$. As is well known, the zero-shear viscosity is governed by the relaxation intensity and relaxation time. ${ }^{12)}$ On the basis of the relaxation spectra of the samples, the higher value of $\eta_{0}$ for LM26 is considered to be because LM26 has larger relaxation intensity as well as longer relaxation time than LM27, although we do not show them here.

This behavior, not seen for regular stars, must be characteristic of the multi-arm stars. Pakula et al. ${ }^{9-11)}$ reported that multi-arm stars exhibit long-time relaxation due to rearrangement of the star molecules having a liquid-like order. The long-time relaxation found for our star PS may have the same molecular origin. However, the relaxation mechanism of the long-time relaxation, and the effects of the number and length of arms on the relaxation have not been fully elucidated yet, as stated in Introduction. Further studies are required for the detailed discussion of the long-time relaxation.

\section{CONCLUSIONS}

The multi-arm star polystyrene samples were synthesized and the dynamic viscoelastic properties of the melts of the samples were investigated. The master curves of storage modulus $G$ ' for the multi-arm stars showed a shoulder at lower frequencies, suggesting that a new mechanical relaxation exists in the long time region where the entanglement relaxation is completed. The multi-arm star polymers also showed a viscosity enhancement compared with the regular stars. The effect of the number of arms on viscosity was observed for the multi-arm star polystyrenes.

\section{ACKNOWLEDGEMENT}

We are grateful to Prof. H. Watanabe and Prof. K. Osaki, Institute for Chemical Research, Kyoto University, for kind guidance in sample preparation and useful comments to this work.

\section{REFERENCES}

1) Fetters LJ, Kiss AD, Pearson DS, Quack GF, Vitus FJ, Macromolecules, 26, 647 (1993).

2) Graessley WW, Roovers J, Macromolecules, 12, 959 (1979).

3) Masuda T, Ohta Y, Kitamura M, Saito Y, Kato K, Onogi S, Macromolecules, 14, 354, (1981).

4) Milner ST, McLeish TCB, Macromolecules, 30, 2159 (1997).

5) Masuda T, Ohta Y, Yamauchi T, Onogi S, Polymer J, 16, 273 (1984).

6) Ohta Y, Masuda T, Onogi S. Polymer J, 18, 337 (1986).

7) Stellbrink J, Abbas B, Allgaier J, Monkenbusch M, Richter D, Likos CN, Löwen H, Watzlawek M, Progr Colloid Polym Sci, 110, 25 (1998).

8) Stellbrink J, Allaier J, Monkenbusch M, Richter D, Lang A, Likos CN, Watzlawek M, Löwen H, Ehler G, Schleger P, Progr Colloid Polym Sci, 115, 88 (2000).

9) Vlassopoulos D, Fytas G, Roovers J, Pakula T, Fleischer G, Faraday Discuss, 112, 225 (1999).

10) Pakula T, Vlassopoulos D, Fytas G, Roovers J, Macromolecules, 31, 8931 (1998).

11) Vlassopoulos D, Pakula T, Fytas G, Roovers J, Karatasos K, Hadjichristidis N, Europhys Lett, 39, 617 (1997).

12) Ferry JD, "Viscoelastic Properties of Polymers", 3rd Ed, Willy, New York (1980). 